\section{Estudio sobre los fundamentos epistemológicos de la administración}

\author{
Study on the epistemological foundations of the \\ administration
}

\section{RESUMEN}

La epistemología está vinculada directamente a la Ciencia, a la Investigación Científica, a la Administración y a la Filosofía. En universidades altamente profesionalizantes propias de nuestra sociedad, en las que la producción de la Ciencia y Tecnología a través de la Investigación Científica, no es necesariamente misión prioritaria, la epistemología resulta siendo aún ajena al quehacer universitario, a cuya consecuencia no existen estudios sostenidos sobre ella y sus conceptos, por regla general, son aún ambiguos y distorsionados.

Las sociedades del nuevo siglo y milenio necesitan una nueva universidad entendida como un centro eminentemente de producción de la Ciencia y Tecnología a través de la Investigación Científica, y sobre esa base, formadora de un nuevo tipo de profesionales competentes para el desarrollo. Esta nueva universidad requiere nuevos planteamientos sobre la epistemología para promover la Investigación Científica, la enseñanza de la ciencia, la Administración de base científica y la Filosofía.

Palabras claves: Epistemología; Epistemología de la Administración; Filosofía

\begin{abstract}
Epistemology is directly linked to Science, Scientific Research, Administration and Philosophy. In highly professional universities of our society, where the production of Science and Technology through Scientific Research, is not necessarily a priority mission, epistemology is still unconnected with university work, which results there are no sustained studies on it and its concepts, as a rule, are still ambiguous and distorted.

The societies of the new century and millennium need a new university understood as an eminently center of production of Science and Technology through Scientific Research, and on that basis, forming a new type of competent professionals for development. This new university requires new approaches on epistemology to promote Scientific Research, Science Teaching, Science-based Administration and Philosophy.
\end{abstract}

Keywords: Epistemology; Epistemology of the Administration; Philosophy.

\section{Nemesio Espinoza Herrera} nespinozah@hotmail.com

Universidad Nacional Mayor de San Marcos, Facultad de Ciencias Administrativas

(c) Los autores. Este artículo es publicado por Gestión en el Tercer Milenio de la Facultad de Ciencias Administrativas de la Universidad Nacional Mayor de San Marcos. Este es un artículo de acceso abierto, distribuido bajo los términos de la licencia Creative Commons Atribucion- No Comercia_Compartir Igual 4.0 Internacional. (http://creativecommons.org/licenses/by-nc-sa/4.0/) que permite el uso no comercial, distribución y reproducción en cualquier medio, siempre que la obra original sea debidamente citada. 


\section{INTRODUCCIÓN}

El presente artículo tiene el propósito de dilucidar tres asuntos vinculantes: la Investigación Científica en Administración, la epistemología de la Administración y la relación de la Administración con la filosofía. La explicación de la referida interrelación permite establecer, aunque de manera preliminar aún, las bases epistemológicas de la Administración $y$, estando la ciencia relacionada a la filosofía, permite también establecer la relación de la Administración con la filosofía. De lo que se trata, por consiguiente, es fundamentar de manera preparatoria el carácter científico y filosófico de la Administración.

En una sociedad como la nuestra, en la que no se da importancia a la Ciencia y Tecnología $\mathrm{y}$ en la que está vigente vigorosamente el concepto de universidad profesionalizante y acientífica, la Administración, tanto en su aspecto teórico y práctico, no solo está estancada sino es extranjerizante y gregaria. Es más, la Administración sigue constreñida a los estrechos marcos organizacionales (empresas e instituciones) mellando su trascendencia en el proceso del desarrollo de la humanidad. En tal contexto, resulta imposible lograr calidad en la formación de los administradores profesionales con niveles adecuados de empleabilidad e imposible contribuir al avance de la Ciencia y Tecnología en Administración.

A pesar de la importancia estratégica que tiene para la Administración los temas del presente artículo, sin embargo, no existe aún una literatura científica ni un programa de investigación acerca de los temas planteados, por lo que es necesario que las tesis de bachiller, de maestría y doctorado, así como las publicaciones científicas, aborden sobre los asuntos relacionados al estatus epistemológico y filosófico de la Administración.

La realidad descrita, demanda con premura, la reestructuración de las universidades y de las facultades de Administración y, dentro de ellas, la de los programas de maestría y doctorado en Administración, con la finalidad -en su sentido teleológico- de que la Administración y la formación de los administradores profesionales estén fundadas necesariamente en la
Investigación Científica, en la epistemología, en la Ciencia y en la Filosofía.

\section{METODOLOGÍA DEL ESTUDIO}

Teniendo en consideración que el tema del estatus espistemológico y filosófico de la Administración es aún agenda pendiente de la Investigación Científica en Administración,por cuya razón la literatura científica acerca de él está en proceso de construcción, significa que se trata de una investigación teórica, de carácter descriptivo y exploratorio. En tales condiciones, la metodología del estudio ha consistido en la recopilación, lectura, análisis de la escasa bibliografía relacionadaa la epistemología, a la investigación científica y a la filosofía.

\section{RESULTADOS}

\section{La universidad}

El concepto trillado que está vigente es que la universidades una institución de "enseñanza superior" que "forman profesionales", tiene "fines" o "misiones", promueve la cultura ylos valores, hace proyección social, etc. etcétera. De manera marginal se dice que la universidad, además de formar profesionales, "investiga". Por otro lado, se señala también que la universidad es una institución que tiene por misión la enseñanza de las profesiones y la investigación científica (Ortega, 1968).

El arraigo de este arcaico concepto acerca de la universidad hace explicable de por qué nuestras universidades no aparecen sostenidamente en el Ranking mundial de las mejores universidades, no tienen patentes, no existe producción científica y tecnológica en niveles y calidades que los tiempos actuales exigen, son "cajas de resonancia" de ciencias y tecnologías foráneas, no existe calidad profesional, se mantiene vigente la ortodoxa metodología "enseñanza-aprendizaje" con mitos, ritos y estereotipos propios de una universidad tradicional, prolifera la pseudoacreditación y, como consecuencia, no existe una efectiva contribución de la universidad al proceso de desarrollo de la sociedad.

\section{La Administración}

La Administración es el proceso de previsión, organización, dirección, coordinación y control (Fayol, 1993). Aunque con un lenguaje 
modernizado y concordante con la condiciones actuales, el anterior concepto acerca de la Administración, sigue siendo, en esencia, el mismo, un siglo despúes.

LaAdministracióin, concebidaasí,se "enseña" en las universidades (tradicionales) y se "forman" profesionales en Administración a la antigua usanza, con un lenguaje y literatura extranjera, principalmente proveniente de Norteamérica. En tales condiciones, la Administración y la formación profesional de administradores están de espaldas a la Ciencia y Tecnología, a la Investigación Científica; a cuya consecuencia, ni la Administración como carrera profesional, ni los administradores "profesionales" no contribuyen, en condiciones en que debían hacerlo, al desarrollo de la sociedad.

La Administración así concebida y practicada, se imparte inclusive en los programas de maestría y doctorado en Administracón, más por razones económicas y de cursilería, sin que haya niveles de desarrollo teórico, científico y filosófico de la Administración. La Administración aún no es objeto de la Filosofía.

\section{Epistemología de la Administración}

En una sociedad en la que no se da importancia a la Ciencia y Tecnología, la epistemología es poco conocida en la univeridad de corte tradicional y, en el caso de la Administración, el asunto de la epistemología es completamente ajena. Revísese las estructuras curriculares de las universidades, en sus facultades no se encontrarán líneas ejes de enseñanza y aplicación de la epistemología, e inclusive es usual que los mismos universitarios (docentes y estudiantes) aborrezcan la epistemología.

Sialgo sehabla acerca dela epistemología en algunas facultades de las vigentes universidades ortodoxas, empero, pervalecen conceptos, por regla general, distorsionados, dogmáticos, gregarios y siempre tales concepciones acerca de la epistemología en el ámbito de las ciencias sociales están sesgadas hacia la epistemología de las ciencias naturales (Hempel, 1983). Hasta se dice que la epistemología es sinónimo de filosofía de la ciencia, evidenciando distorsiones en la naturaleza de la epistemología y de la filosofía (Moulines, 2011).
Así, pues, la epistemología per sey la epistemología de la Administración en particular son asuntos ajenos al quehacer en la "formación profesional" de los administradores en las universidades tradicionales. Sin embargo, las sociedades de hoy (sociedades del conocimiento) exigen el desarrollo de la epistemología universal en general y de la epistemología de la Administrción en particular (Hernández, 2006). Demanda, por lo tanto, la divulgación de la necesidad de la relación de sujeto y objeto de la investigación en el ámbito de la Adminstración (Flores, 2013) en el sentido de que el investigador (sujeto) esté vinculado a la Administración (objeto). Demanda también establecer el carácter científico de las ciencias sociales, por lo tanto, de la Administración (Gellner, 1984).

Así como se plantea que las otras disciplinas -Educación, por ejemplo, (Barriga, 2007)- tienen fundamentos espitemológicos que les otorga su carácter científico, también en Administración, aunque de manera aún preliminar, se viene cuestionando si ella tiene su carácter epistemológico, vale decir, su carácter científico (Bunge, 1993).

\section{La Investigación Científica en Administración}

En las universidades tradicionales la investigación científica, en lugar de ser prioritaria, es marginal. En el caso de la Administración, la verdadera investigaión científica es virtualmente inexistente por la primacía aún del nefasto "bahillerato automático" con el que se gradúan a los "administradores" sin realizar labores de investigación científica; en maestría en Administraciónse graduan con tesis que no reunen las condiciones epistemológicas (científicas),y en el doctorado en Administración con tesis sin filosofía.

Es más, la investigación científica en Administración -al igual que en el caso de las otras disciplinas profesionales del ámbito de las ciencias sociales- las precarias investigaciones se hacen a usanzas arcaicas de la epistemología propia de las ciencias naturales (De la Garza, 2010) 


\section{La filosofía y la Administración}

La filosofía en nuestro medio, propio de sociedaes subdesarrolladas,así como en el caso de la ciencia, aún no forma parte de la cultura nacional. Enclaustrada en pequeños círculos y solo en la facultad o escuela de filosofía, la filosofía está cargada de dogmatismos, gregarismos, oscurantismos, ambigüedades, presunciones e intolerancias; $\mathrm{y}$, en tales condiciones, la filosofía es ajena al proceso del desarrollo de la sociedad. La Filosofía está, pues, de espaldas a la población y alejada de su principal motivo e inspiración: el bienestar de la humanidad.

Siendo la epistemología, la investigación científica, la ciencia y la tecnología asuntos desvinculados a la Administración, resulta fácilmente explicable de por qué en el ámbito de la Administración, la filosofía es completamente extraña a ella y de cómo la Administración no está concebida en las complejidades sociales (Snowden-Stanbridge, 2012). En el programa de doctorado en Administración, un programe eminentemente de base filosófica, por cuya razón no existe, por ejemplo, tesis relacionadas a la filosofía y la Administración. Cuanta falta hace la filosofía en el contexto de la Administración para comprender la trascendencia universal de ella en la búsqueda de las aproximaciones hacia la entelequia de la felicidad humana.

\section{DISCUSIÓN}

¿Qué es la universidad? Muy distinto al concepto tradicional de universidad que concibe a ella como un centro eminentemente de formación de profesionales, la verdadera universidad es un centro eminentemente de producción de la Ciencia y Tecnología a través de la Investigación Científica-cuyo eje es la epistemología-, y sobre esa base, la universidad forma profesionales competentes. En tal sentido, la universidad tiene dos misiones ejes, fundamentales, simbióticas; y en este orden: 1) la producción de la Ciencia, Tecnología e Innovación a través de la Investigación Científica y, sobre esa base, 2) la formación de un nuevo tipo de profesionales para el desarrollo.

En sociedades propias de un nuevo siglo y milenio como la que transita actualmente la humanidad, la díada Ciencia-Tecnología - cuya fuente es la Investigación Científica- es el principal soporte del desarrollo económico y social; $y$, en tales condiciones, la universidad tiene que prioritariamente realizar investigaciones científicas para producir o crear Ciencia y Tecnología, y sobre esa base -si y solo si, sobre esa base-, formar profesionales de calidad.

Sin embargo, lo que tenemos son universidades altamente profesionalizantes y acientíficas por cuanto se limitan prioritariamente a "forman profesionales" de espaldas a la Investigación Científica, a la epistemología y están inmersas -por regla general- en un contexto de la consuetudinaria corrupción y mediocracia. En tales condiciones, las universidades vigentes no producen Ciencia y Tecnología, es inexistente la enseñanza de la epistemología y están imposibilitadas a formar profesionales competentes de calidad. Así, pues, lo que tenemos son pseudo-universidades.

Estas consideraciones plantean la imperativa necesidad de la reestructuración integral y sostenida de las universidades a fin de lograr -a mediano y largo plazo- que ellas sean centros eminentemente de Investigación Científica, de producción de la Ciencia y Tecnología, de enseñanza de la epistemología; $y$, sobre estas bases, sean formadoras de profesionales competentes de nuevo tipo y sean universidades con una gestión proba y eficiente.

En las condiciones anteriormente descritas, las universidades eminentemente profesionalizantes y acientíficas que tienen facultades de Administración "forman bachilleres y profesionales" en Administración no necesariamente competentes -y hasta gradúan a masters y a doctores en Administración-, irremediablemente a usanzas tradicionales y de espaldas a la Ciencia y Tecnología por cuanto no existe Investigación Científica propiamente dicha y el asunto de la epistemología, por ejemplo, es un asunto virtualmente proscrito y se mantiene vigoroso aún el espíritu del "bachillerato automático"; $y$ en "posgrado" -programas altamente profesionalizantes y acientíficas, al igual que en "pregrado"-, las tesis en Administración son, por regla general, intrascendentes sin aporte real a la Ciencia y Tecnología en el ámbito de la Administración. 
En los albores del Siglo XXI y del Tercer Milenio, las sociedades demandan una nueva Administración de base científica, por consiguiente, demandan la reestructuración de las facultades de Administración que posibilite que en ellas exista prioritariamente la producción de la Ciencia y Tecnología en el ámbito de la Administración -en pregrado, $\mathrm{y}$ especialmente en posgrado- $\mathrm{y}$ haya formación de un nuevo tipo de profesionales en Administración, competentes y para el desarrollo.

Esto quiere decir que la Administración es una ciencia porque tiene un conjunto de conocimientos científicos con los cuales se explican, se interpretan y se argumentan satisfactoriamente los fenómenos -que son hechos humanos intencionales- en el ámbito de la Administración.

El hecho de que existan conocimientos científicos acerca de la Administración planteados por muchos investigadores (Taylor, Fayol, McGregor, Mayo, Maslow, Lewin, Jiménez Nieto, Luthans, Hammer, Deming, Kast, Mintzberg, Simon, Senge, Weber, Porter, Hofstede, Drucker, Peters, Hamel, Kliksberg, Bunge, Rafael García Castillo, etcétera); evidencian que la epistemología de la Administración existe y está en proceso de construcción en concordancia con los avances de la Ciencia y Tecnología, con cuyo hecho existen fundamentos para el replanteamiento de la teoría de la Administración en el marco de la trascendencia que tiene la ciencia y tecnología para reivindicar a la Administración como el factor principal del desarrollo económico y social de las sociedades modernas.

Así, pues, resulta necesario admitir que la Administración tiene fundamentos epistemológicos (científicos) y filosóficos. Esto quiere decir que la Administración tiene el estatus epistemológico (Bunge, 1993). En otras palabras, la Administración es Ciencia.

¿Qué es la epistemología? Es el tratado del conocimiento científico. Y ¿qué es la Ciencia? es el conjunto de conocimientos científicos. La epistemología, por lo tanto, es el tratado o estudio de la Ciencia. En otras palabas, la epistemología es el estudio del conocimiento científico. En este contexto, el planteamiento consiste en que la Administración está fundada en los conocimientos científicos, en la Ciencia; por consiguiente, la Administración tiene fundamentos epistemológicos, científicos -y, como más adelante se planteará, también filosóficos. Sobre el carácter científico de la Administración es necesario admitir que está en discusión en los ámbitos académicos y existe aún diversos obstáculos epistemológicos para tal reconocimiento (Borgucci, 2012).

He aquí la principal razón de por qué es necesario hablar acerca de la epistemología de la Administración que no es sino el tratado del conocimiento científico o de la ciencia de la Administración lo que conlleva a plantear, categóricamente, que la Administración es Ciencia y debe ser tratada como tal. La epistemología de la Administración, por las razones explicadas y especialmente por el carácter profesionalizante de las universidades vigentes, es aún embrionaria (Garcíacastillo, 1997). El desarrollo de la Administración teórica y práctica está supeditado al nivel de desarrollo de su epistemología.

Las anteriores consideraciones conllevan a abordar el asunto de la Investigación Científica, especialmente al de la Investigación Científica en Administración. ¿Qué es la Investigación Científica? Es una actividad distintiva y prioritaria de la universidad que consiste en producir Ciencia y Tecnología. Producir Ciencia y Tecnología significa crear nuevos conocimientos científicos, tecnologías e innovaciones. La Investigación Científica en Administración-fundada en la epistemología de la Administración- significa, por consiguiente, que las facultades de Administración deben producir o crear Ciencia, Tecnología e Innovación en Administración.

Si una investigación científica emprendida en el ámbito de la Administración no produce nuevos conocimientos, nuevas tecnologías e innovaciones, o si los productos finales de la Investigación (artículos científicos, tesis, ponencias, libros, etc.) no contribuyen realmente al avance de la Ciencia y Tecnología en Administración, entonces no es una verdadera investigación científica, sino conato de ella; es una pseudo-investigación. Por eso es que existe la necesidad de discutir el asunto 
de la epistemología de la Administración para desarrollar la Investigación Científica en el ámbito de la Administración (Vargas, 2013).

Las aseveraciones de los párrafos anteriores plantean la imperativa necesidad de la reestructuración de las facultades de Administración, especialmente en cuanto a su estructura curricular en la que haya desde el primer Ciclo hasta el final- el eje de la Investigación Científica que garantice la enseñanza de la Ciencia, de la epistemología y del proceso de creación científica y tecnológica en Administración, que garantice la aprobación de tesis de trascendencia para graduar a los bachilleres. Igualmente existe la necesidad del proceso de reestructuración integral de los programas de maestría y doctorado en Administración que son, al igual que pregrado, programas altamente profesionalizantes, sin fundamentos epistemológicos (HurtadoRivera, 2008).

Ahora bien. Los asuntos tratados acerca de la universidad, epistemología e investigación científica vinculados a la Administración, conllevan abordar otro asunto: la relación de la Administración con la Filosofía. La Filosofía es la reflexión -basada en los conocimientosacerca de la existencia humana y de su relación a todo cuanto hay en el mundo y en el infinito Universo. El término reflexión -palabra clave para la Filosofía- admite sinónimos: admiración, contemplación, meditación, pensamiento, entendimiento, comprensión, percepción, discernimiento, aprehensión, juicio, interpretación.

El anterior concepto acerca de la Filosofía permite hacer tres planteamientos: 1) La filosofía está basada en la Ciencia, vale decir, en los conocimientos científicos. Ciencia y Filosofía son vinculantes, simbióticas (Bunge, 1966);2) La Filosofía es única, transdisciplinaria. La Filosofía, muy contrario a lo que convencionalmente se dice (Bunge, 2007), no es disciplinar, en tanto que la epistemología sí los es. Por consiguiente, es apropiado hablar de epistemología de la Administración, epistemología de la economía, epistemología de la educación, etcétera. Empero, no es apropiado hablar de Filosofía de la Administración, Filosofía de la economía, Filosofía de la educación, Filosofía de la lógica, etc., como si cada disciplina tuviera su propia filosofía, como en lugar de una, hubiera muchas filosofías.

Por la anterior razón es que no se habla -no debía hablarse- de Filosofía de la Administración, sino más bien, de la vinculación de la Administración con la Filosofía; es decir, de la Filosofía y Administración; y, 3) La epistemología no es "filosofía de la ciencia"como frecuentemente se hace entrever (Bunge, 2007).Los mismos conceptos de filosofía y epistemología explican de por qué esta última no debe ser considerada como "filosofía de la ciencia".

Así, pues, cuando se habla acerca de los temas vinculantes (universidad, Administración, epistemología, ciencia, investigación científica) es necesario engarzar con la Filosofía porque es en el contexto de ella la que se percibe la trascendencia ecuménica de la Administración como factor clave para el desarrollo de la humanidad, desarrollo que en esencia significa calidad de vida para el ser humano con la visión de disminuir grandemente el sufrimiento y la aproximación hacia mayores niveles del ideal de la felicidad humana. No obstante, ni en las facultades de filosofía se habla acerca de la Administración, ni en las de la Administración se habla acerca de la Filosofía. En buena cuenta, la Filosofía deja de ser tal, si en el proceso de la reflexión no está presente la Administración. La Administración, por su lado, es mellada y restada de importancia en cuanto a su trascendencia en el desarrollo de la humanidad, si no está inmersa en la Filosofía.

En concordancia con lo expuesto, y con la finalidad de apuntalar al desarrollo de la relación de la Administración con la Filosofía, es que se percibe la necesidad de un proceso de reconversión de los programas de doctorado en Administración, que no son programas profesionalizantes ni de investigación científica propiamente dicha como es usual en nuestra realidad, sino son -deben ser- programas eminentemente de reflexión trascendental acerca de la Administración.

Los programas de doctorado en Administración ya no son de Investigación Científica sino de reflexión sobre la trascendencia filosófica de la Administración. La distinción entre un programa de maestría 
y un programa de doctorado es que el primero es de investigación científica de máximo nivel y el segundo es de Filosofía, y tiene por finalidad de construir un pensamiento filosófico sobre la trascendencia de la Administración en el desarrollo de la humanidad. El doctor en Administración ya no es un científico como sí lo son los bachilleres y los magísteres en Administración. El doctor en Administración es un filósofo.

El grado académico de doctor en Administración no es, por consiguiente, un asunto cursi, sino una acreditación de que se concibe a la Administración en su trascendencia filosófica. Precisamente por estas razones existimos una plétora de "doctores en administración" sin pensamientos filosóficos y sin ubicar a la Administración en su real dimensión en los destinos de la humanidad en este mundo y en el infinito Universo.

\section{CONCLUSIONES}

1. Las universidades vigentes son altamente profesionalizantes y acientíficas porque "forman profesionales" de espaldas a la Ciencia y Tecnología, al margen de la Investigación Científica, de la epistemología. Por esta razón es que no hay producción científica y tecnológica en términos que los tiempos actuales exigen, ni tampoco existe calidad profesional. Todo esto demanda la reestructuración universitaria para lograr que la universidad sea un centro eminentemente de producción de la Ciencia y Tecnología a través de la Investigación Científica y, sobre esa base, formadora de un nuevo tipo de profesionales para el desarrollo nacional.

2. La Administración tiene el estatus epistemológico por cuanto se fundamenta en los conocimientos científicos. Es decir, la Administración tiene carácter científico. En otras palabras, la Administración es Ciencia.

3. La epistemología de la Administración está en proceso de construcción y demanda la necesidad de áreas, programas, líneas de la Investigación Científica en Administración. La formación de los profesionales en Administración, por el carácter científico de la Administración, debe estar supeditada a la Investigación Científica en Administración, entendida a ésta como la producción de la Ciencia y Tecnología en el ámbito de la Administración. Todo esto demanda la necesidad nacional de establecer procesos sostenidos de reestructuración curricular en Administración cuyo eje sea la Investigación Científica que garantice la enseñanza de la epistemología, es decir, la enseñanza de la Ciencia.

4. Estando la Administración fundada en la epistemología, la investigación científica en Administración está expresada en dos niveles: A)La Investigación Científica básica con la formación de investigadores científicos en Administración acreditada con la aprobación de una tesis de bachiller de trascendencia, con aportes al avance de la Ciencia y Tecnología en Administración; y, B) La Investigación Científica en Administración de máximo nivel científico a través de los programas de maestría en Administración acreditada con la aprobación de una Tesis de maestría de trascendencia, de alta rigurosidad científica y con aportes reales a la Ciencia y Tecnología en Administración. El programa de doctorado en Administración ya no es de Investigación Científica sino de construcción de teorías y pensamientos acerca de la trascendencia filosófica de la Administración.

5. Entre Ciencia y Filosofía existe una vinculación simbiótica. No hay filosofía sin ciencia, y ésta sin la primera queda mediatizada su trascendencia en el proceso del desarrollo de la humanidad. La Filosofía es la reflexión -basada en los conocimientos científicos-acerca de la existencia humana y de su relación a todo cuanto hay en el mundo 
y en el infinito Universo. Por estas consideraciones la Administración como Ciencia debe trascender hacia el ámbito de la Filosofía. Así, pues, la Administración está -debe estarestrechamente relacionada a la Filosofía.

\section{REFERENCIAS BIBLIOGRÁFICAS}

1. Barriga Hernández Carlos (2007). Acerca del carácter científico de la pedagogía. Tesis para optar el grado de doctor en Educación. Disponible en: http://cybertesis.unmsm.edu. pe/bitstream/cybertesis/2617/1/Barriga_ hc.pdf.

2. Borgucci, Emmanuel (2012). Algunos problemas epistemológicos de las ciencias administrativas. Revista del Centro de investigación de Ciencias Administrativas y Gerenciales. CICAG, VOL 9, №. 2. Universidad Privada Dr. Rafael Belloso Chacín (URBE), Maracaibo, Estado Zulia, Venezuela. Disponible en http://publicaciones.urbe.edu/index.php/ cicag/article/viewArticle/1185/3294

3. Bunge Mario (1993). Status epistemológico de la administración J. J. Ader. Buenos Aires, Paidos. Argentina. Documentos en Word y en PDF. Disponible en http://aulavirtual.agro.unlp. edu.ar/pluginfile.php/21410/mod_resource/ content/2/bunge_status_epistemologico_ administracion.pdf

4. Bunge Mario (2007). Epistemología. Editorial Ariel SA. España.

5. Bunge Mario (1966).La ciencia, su método y su filosofía. Ediciones Siglo XX. Argentina.

6. De la Garza Enrique y Leyva Gustavo, coordinadores (2010). Tratado de metodología de las ciencias sociales; perspectivas actuales. Libro electrónico disponible en: https://drive.google.com/file/ d/0B00rfQ9umQlAT0hhWUpLMHZDOXc/view

7. Fayol Henry (1993). Administración general e industrial. Editorial Universitas. Lima - Perú.

8. Flores Jiménez Ivette (2013) La Teoría del conocimiento y la epistemología de la administración. Boletín CientíficoXIKUAVol $1 \mathrm{~N}^{\circ}$ 1. Universidad Autónoma del Estado de Hidalgo México. (Ruth Flores Jiménez, Juan José Aguilar
Lugo Marino). Disponible en www.uaeh.edu. $\mathrm{mx} / \mathrm{scige}$

9. Garcíacastillo y Cruz Rafael (1997). Epistemología de la Administración; propuesta para la formación de los administradores. Artículo en PDF disponible en http://www. econ.unicen.edu.ar/attachments/113_ej25.pdf

10. Gellner Ernest (1984). El rango científico de las ciencias sociales. Revista internacional de Ciencias Sociales. UNESCO. Disponible en www.unesdoc.unesco.org/ images/0006/000636/063623so.pdf

11. Hempel Carl G. (1983). Filosofía de la Ciencia Natural. Alianza Editorial. España.

12. Hernández Iglesias José (2006). Hacia una epistemología de la administración en un mundo globalizado. Revista Suma Administrativa Vol $1 \mathrm{~N}^{\circ} 1$. Bogotá, Colombia. Disponible en www. konradlorenz.edu.co/images/stories/suma_ administrativa/200611/hacia.pdf

13. Hurtado Eulogio - Rivera Félix (2008). El requerimiento del marco epistemológico en las tesis de posgrado. Revista Gestión en el Tercer Milenio UNMSM Vol $9 \mathrm{~N}^{\circ} 1$.

14. Moulines Carlos Ulises (2011). El desarrollo moderno de la Filosofía de la Ciencia (18902000). Instituto de Investigaciones Filosóficas. Universidad Nacional Autónoma de México. México.

15. Ortega y Gasset José (1968).Misión de la Universidad. Colección Arqueros. Editorial Revista de Portillo Zoraida. España.

16. Snowden David - Stanbridge Peter. (2012).The landscape of management: creating the context for understanding social. Documento en PDF disponible en: http://citeseerx.ist.psu.edu/viewdoc/ download?doi=10.1.1.452.1904\&rep=rep1\& type $=$ pdf

17. Vargas Hernández José (2013).Epistemología dialógica de las ciencias administrativas. Cuadernos Latinoamericanos de Administración. Volumen IX, Número 17. (Adrián de León Arias - Andrés Valdez Zepeda Carmen Leticia Borrayo). 